\title{
Una familia en formación con muchas dificultades
}

\section{RESUMEN}

Se presenta el caso de una pareja joven con una hija con conflictos entre ellos y con sus familias y en la que la joven esposa sufre un serio trastorno emocional. Esta familia destaca por las dificultades con las que afrontan las tareas de esta etapa del ciclo vital. Estas dificultades vienen determinadas principalmente por los problemas previos de la joven en su familia a raíz de la muerte de su padre, así como al modo como han establecido su relación: "quemando etapas", de modo que sin apenas noviazgo ya están casados y con una hija.

Tomando como guía el marco teórico del ciclo vital el médico de familia puede intervenir para reconducir la dinámica de la relación de la pareja y modificar su clima. Esto no significa que dada la magnitud de las tareas no sea necesario un trabajo a largo plazo en el nivel secundario que el médico de familia puede contribuir a iniciar.

Palabras clave: Medicina de Familia. Dinámica familiar. Atención Familiar. Ciclo vital. Duelo.

\section{A formating family in difficulties}

\begin{abstract}
The case of a young couple with a child is presented, where important conflicts between themselves and with their families have emerged and in which the wife suffers from acute emotional disturbance. This family faces many difficulties in accomplishing the relevant developmental tasks at this stage of their life cycle. These difficulties are mainly due to earlier problems in the wife's own family set off by her father's death and subsequently by the way that this family begin their life together, "jumping" phases of the life cycle. They marry and have a child with hardly any preparatory time spent previously together.

The family doctor, taking the life cycle framework as a guide, can intervene to redirect the couples' relationship and improve their emotional climate. Given the magnitude of the task, longterm specilized help should be expected to be carried out and which the family doctor can help to initiate.
\end{abstract}

Key words: Family Medicine. Family dynamic. Family Health Care. Life cycle. Bereavement.

\section{INTRODUCCIÓN}

Entre los médicos comprometidos con una orientación psicosocial se hace necesario en muchas situaciones habituales de la consulta, pensar en términos de familia ${ }^{1}$. A veces en estas situaciones la implicación familiar se sitúa en el trasfondo y muchos médicos deciden mantenerse en nivel in- dividual. Por el contrario, en otras ocasiones en las que el conflicto familiar aparece en primer plano, la tarea de redefinir la demanda del paciente en términos de sistema familiar es más fácil. Sin embargo, estos casos pueden ser muy complejos y precisan derivación al nivel especializado, si bien esto no significa que el médico de familia no tenga un importante papel a realizar. 
Cuando un paciente acude por la existencia de una crisis en las relaciones familiares, como ocurre en la mayoría de problemas psicosociales, es útil para evaluar el problema y definir la intervención más apropiada, recurrir a analizar la etapa del ciclo vital familiar en la que se encuentra el paciente y determinar si las tareas propias de esta fase están siendo adecuadamente afrontadas y resueltas. El ciclo vital, un marco teórico que concibe a las familias como un conjunto de personas que viajan juntos en el tiempo, ofrece un modelo para la descripción de la "patología familiar" y una base para planes terapéuticos ${ }^{2}$. El ciclo vital permite al médico de familia desarrollar acciones preventivas y generar hipótesis sobre las posibles fuentes de disfunción familiar ${ }^{3}$.

El caso que presentamos es el de una familia joven que acaba de tener su primer hijo. Con una corta fase de noviazgo y sin haber pasado por el periodo del nido sin usar (pareja que ha formado un hogar y todavía no ha tenido hijos), su inicio como familia no está resultando fácil y están encontrando problemas para completar exitosamente las tareas de la fase del ciclo en el que se encuentran. Cada fase del ciclo vital tiene unas serie de tareas específicas que se deben alcanzar para satisfacer las necesidades de sus miembros ${ }^{4}$. Los problemas y las tareas no superadas de etapas previas pueden bloquear el comienzo de una nueva fase al acumularse con las nuevas ${ }^{5}$. Esto es lo que le ocurre a la familia que presentamos: afrontan el desafío de ser padres sin haber superado las fases previas y sin haber terminado el proceso de individualización en sus familias de origen.

\section{PACIENTE IDENTIFICADO Y MOTIVO DE CONSULTA}

Noelia de 22 años es remitida desde la consulta de pediatría, donde ha acudido con su hija de 27 días, porque se descubren conflictos entre Noelia, su pareja Manuel de 24 años y la madre de Noelia, Camino de 59 años. El bebé es alimentado con lactancia materna, no muestra ningún síntoma, crece bien, duerme bien, su comportamiento es normal. Noelia fue vista por un médico de urgencias hace unos días y le fueron prescritos un antidepresivo y una benzodiacepina. Ella ha decidido no tomar el tratamiento porque no quiere dejar de lactar. Manuel opina que lo primero es ella y que si hace falta que deje la lactancia la debe dejar, la madre de Noelia también comparte esta opinión. Tanto el comportamiento de Noelia como el de Manuel, que se muestra contento y orgulloso de su hija, indican que ambos están muy comprometidos con su cuidado. También Camino, la abuela, ha aceptado a su nieta y la cuida con cariño. A la consulta acuden los dos miembros de la pareja y la niña. Ambos manifiestan que Noelia pierde los nervios con frecuencia y se pone como "loca" principalmente con su madre, aunque últimamente también con Manuel. Sin embargo con su hija se mantiene siempre tranquila. Noelia se siente triste y piensa que los vecinos la observan y critican a sus espaldas. Apenas mantiene relaciones sociales al margen de las familiares.

El genograma de esta familia se presenta en la figura 1 .

\section{HISTORIA DEL PROBLEMA}

Noelia y Manuel se conocieron hace 1 año y a los 3 meses Noelia se quedó embarazada. A pesar de que Noelia no tenía menstruaciones, no se "percartaron" del embarazo hasta que Noelia estaba de 6 meses. Aún así mantuvieron el embarazo en secreto para sus familias hasta el día del parto. Pocos días después del nacimiento de la hija de ambos contrajeron matrimonio. Al poco tiempo de conocer a Manuel, Noelia abandonó el domicilio de su familia y se fue a vivir a casa de la familia de Manuel.

Manuel es un joven normal con buena relación con su familia, trabajo fijo en una empresa familiar y una buena relación social. Es el último hijo de una familia de seis hermanos. Por el contrario, Noelia ha tenido una adolescencia muy difícil desde la muerte de su padre (había sido una buena estudiante hasta entonces) hace 7 años. Cuando esto sucedió se trasladaron desde otra provincia a donde ahora residen. Camino, la madre de Noelia padece un cuadro ansioso y un trastorno de personalidad,

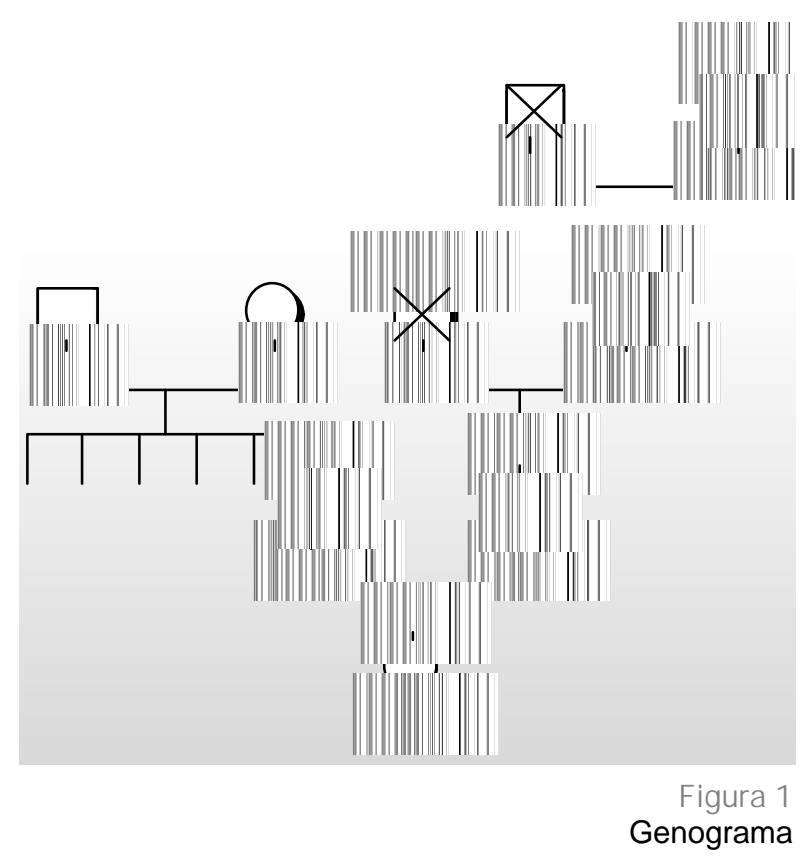


carece de capacidad de contención emocional, tiende a agobiarse y a arruinar las posibilidades de apoyo que se le ofrecen. La abuela de Noelia ha desarrollado una demencia grave en los últimos tres años. Hasta que Noelia conoció a Manuel se encontraba aislada, sin amistades y no había tenido ninguna pareja. La situación en su casa era caótica y vivía encerrada sufriendo crisis de ansiedad, síntomas depresivos e ideas de referencia. Los conflictos con su madre eran continuos con violencia verbal.

Camino carece de parientes propios cercanos y la relación con la familia de su marido es muy mala debido, entre otras razones, a disputas por la herencia. Toda la familia vivía de la pensión de viudedad que aunque no es miserable tampoco es alta. La familia de Manuel tampoco es de nivel social alto aunque no tienen dificultades económicas. Manuel dejó de estudiar al terminar educación primaria.

Poco después de nacer la niña, Noelia y Manuel se trasladaron desde la casa de la familia de éste a la casa de la madre, con ella y la abuela de ésta. Aunque Noelia manifiesta que lo que quiere es que vivan solos y no con ninguna de las dos familias. Desde entonces los conflictos entre Noelia y su madre han sido continuos; en los últimos días también ha habido discusiones entre Manuel y Camino y entre Noelia y Manuel. La salida de casa de Manuel fue sin motivo concreto aparente y a iniciativa de Noelia que sentía que su familia política se estaba inmiscuyendo demasiado. Manuel dice que sus padres y hermanos están dispuestos a apoyarles en lo que sea y sin condiciones.

La relación entre Manuel y Noelia se ha deteriorado en los últimos tiempos habiendo aparecido conflictos abiertos entre ambos. Manuel a veces también pierde la paciencia con Noelia y la acusa de no poner nada de su parte para curarse, pero en general mantiene una actitud precavida evitando manifestar abiertamente sus sentimientos. Incluso la actitud generalmente comprensiva de él, parece que le exaspera y le hace sentirse más incapaz.

\section{COMENTARIOS SOBRE LA ESTRUCTURA E HISTORIA FAMILIAR}

Se trata de una familia en formación y a la vez con hijos pequeños, sin que haya existido un periodo de noviazgo de una duración suficiente para que los objetivos a alcanzar durante éste (creación de un vínculo afectivo, selección de la persona y compromiso con ésta, inicio de conocimiento mutuo, etc.) se hayan alcanzado. Esta familia se ha encontrado directamente en la etapa II del ciclo vital, habiendo esquivado no solamente la etapa de noviazgo sino también la de nido sin usar. La llegada del primer hijo a la familia produce una crisis que para resolverse requiere que las tareas de las fases anteriores, es decir de la fase de constitución de la pareja, hayan sido abordadas y superadas. Frecuentemente nos encontramos con problemas relacionados con el hecho de "saltar" fases del ciclo vital ${ }^{6}$. Así sabemos que las parejas que se "saltan" las primeras etapas del ciclo vital y empiezan su convivencia en relación con un embarazo, sin haber tenido la oportunidad de separarse emocionalmente de sus padres ni de haber establecido una relación intima entre ellos, tienen un mayor riesgo de presentar disfunciones familiares ${ }^{7}$.

Noelia viene de una situación familiar caracterizada por un desarrollo congelado debido a un duelo no resuelto. La muerte del padre ocurrió cuando la familia avanzaba en la etapa de familia con adolescente y afrontaba la tarea de iniciar la preparación de la salida del hogar de Noelia. El duelo ha imposibilitado este desarrollo necesario, quedando la madre y la hija atrapadas en una relación que impedía el desarrollo normal de la adolescencia de Noelia, una relación fusionada con conflictos en la que los límites paterno filiales quedaban desdibujados. Las pérdidas pueden enlentecer la transformación de los lazos entre las generaciones y convertir la tarea de constituir una nueva familia más difícil para las generaciones jóvenes. Una reacción común es una respuesta de fusión por parte de la familia ${ }^{8}$. El nacimiento de la hija (no está el abuelo) y su boda con Manuel (hubiera sido el padrino) ha podido poner de relieve de nuevo y con fuerza la falta del padre. El duelo de Noelia se realizó sin un mínimo apoyo por parte de su madre, sin capacidad de contención y sin ninguna intervención externa desde la red social o los servicios socio sanitarios que le ayudarán. En estas condiciones su elaboración de duelo ha estado muy obstaculizada ${ }^{9}$. De hecho, la enfermedad terminal y el duelo es un periodo en el que la familia necesita un gran apoyo ${ }^{10}$, esta necesidad de apoyo es aún mayor si hay adolescentes implicados, por el conflicto directo entre la tarea evolutiva primordial de ésta y la experiencia de la pérdida ${ }^{11}$.

Todo esto hace que la pareja ahora empiece su relación desde un punto de partida desigual, ya que mientras Noelia es una persona cargada de problemas y con una mala relación con su familia de ori gen, Manuel ha llevado una vida normal y se encuentra integrado familiar y socialmente. Llama la atención que Noelia haya propuesto la salida de la casa de Manuel y solicite que vivan solos. Aparentemente le hubiera sido más fácil aceptar la ayuda “desinteresada" que la familia de Manuel le ofrecía. Esto es un gesto saludable de Noelia que puede haber intuido que esta ayuda no va a ser tan desinteresada y se va a enfrentar al peligro de intromisiones o incluso ya ha percibido peticiones y presiones poco aceptables. Tenemos que tener en 
cuenta que para una joven pareja tan peligrosas son las censuras no constructivas, como las ayudas exageradas y permanentes ${ }^{12}$. Incluso que la familia de Manuel pueda ejercer un cierto tutelaje económico puede producir una pérdida de independencia (real o al menos subjetiva) en ambos y especialmente en Noelia. No hay que negar la voluntad de colaboración y las buenas intenciones de la familia de Manuel, pero esto puede impedir que la nueva pareja tenga la oportunidad de definir su propia relación y escoger la vida que quieren llevar. La implicación de las familias de origen puede ser causa de futuras desavenencias ${ }^{13}$. Ante futuros problemas y dificultades puede hacerse más difícil la negociación entre la pareja ya que estará siempre la tentación de echar las culpas a otros, en este caso la familia de Manuel y, por el otro lado, éste y su familia pueden acusar a Noelia de ser una desagradecida.

Pero no solamente la ayuda desproporcionada y la intromisión de la familia de Manuel es un peligro. También debemos tener en cuenta que la familia de Noelia ha venido caracterizada por mujeres que enviudan jóvenes con una hija única. Es una estructura en las que las figuras masculinas están excluidas. Manuel no tiene fácil entrar en esta familia, en la que parece que la inercia de la historia familiar no le deja hueco. Si por cualquier razón Manuel se ausentara, la historia volvería a repetirse y Noelia criaría a su hija con la "ayuda" de su madre y sin el padre. Es necesario subrayar que la figura paterna tiene un papel muy importante en el desarrollo psicológico de los hijos ${ }^{14}$. Uno de los peligros que acechan a esta familia es el que se produzca un relación muy estrecha entre madre e hija que no dé entrada al padre. Esta pauta de relación constituye una disfunción habitual de la familia en formación ${ }^{15}$.

Otro dato llamativo en esta historia es que tanto Noelia como Manuel no se "dieran cuenta" del embarazo. Por una parte esto ha significado que se ha perdido otra etapa: la del embarazo que es importante para preparar la vida en común con un hijo y sobre todo para crear el vínculo entre ambos miembros de la pareja (especialmente Noelia) y su futura hija. Esta negación probablemente haya sido un mecanismo de defensa de la pareja para no enfrentarse a la angustia que le podía provocar la conciencia de la situación tan complicada que tenían. Por lo menos, aunque se trate de una etapa perdida, de este modo se ha evitado que el embarazo haya sido una experiencia negativa y llena de angustia. Debemos subrayar que, como hemos dicho, los dos están satisfechos con el nacimiento de su hija y se encuentran comprometidos con su cuidado. Si se hubieran percatado antes del embarazo, mi impresión es que hubieran decidido continuar con él y no optar por una interrupción voluntaria de éste.

La comunicación entre Manuel y Noelia está di- ficultada por la habitual pauta de relación entre el afectado de un trastorno psicológico y sus allega$\operatorname{dos}^{16}$. El afectado por un problema de salud mental no se siente entendido por los que le rodean y éstos viven su sufrimiento como un cierto fracaso personal, ya que no son capaces de motivar al deprimido para que salga adelante. Pero al mismo tiempo, los que le rodean se ven asaltados por sentimientos de agresividad hacia éstos "que no ponen bastante de su parte para mejorar" y se sienten culpables por la posibilidad de herir al paciente. Esto último les puede llevar a no manifestar sus opiniones y preferencias y a ocultar sus sentimientos negativos. Esta contradicción puede ser muy estresante para el allegado. En este caso además la relación se resiente por la incapacidad de Noelia de expresar afecto o comunicar sus sentimientos. Noelia parece mostrar una desconfianza general hacia todo el mundo que intenta acercarse (se explica por la falta de una figura parental en la que apoyarse y quizás malas experiencias que no relata de relaciones abusivas). Esta desconfianza de Noelia hacia todo el mundo también se extiende a Manuel, parece que no se cree que éste le pueda querer de verdad a pesar de sus manifestaciones verbales en ese sentido y su comportamiento cariñoso.

\section{TAREAS}

Para definir el plan de actuación que se nos plantea con esta pareja tenemos que recordar cuáles son las tareas que se plantean en las etapa del ciclo de la familia en la que nace el primer hijo: la asunción del rol parental, la adaptación de las relaciones de la pareja para acomodarse al nuevo miembro, la reformulación de las relaciones con las familias de origen que pasan a la situación de abuelos ${ }^{2}$. Esta etapa está caracterizada en general por un movimiento centrípeto entre las generaciones. Sin embargo, esta pareja se encuentra con estas tareas cuando todavía no ha completado la separación de las familias de origen ni ha definido su vínculo como pareja que definen la etapa previa del ciclo vital. Cuando una pareja se ha saltado las primeras etapas de su ciclo vital, es necesaria una intervención que facilite a la pareja la resolución de las dificultades que supone transitar por las etapas I y II del ciclo vital familiar simultáneamente ${ }^{17}$. Por lo tanto esta compleja situación conlleva definir unos objetivos de intervención profesional que se relacionan con tareas que proceden de diferentes etapas del ciclo vital, así como otras que son consecuencia de la juventud de la pareja y de los problemas heredados del periodo previo a su relación. Podíamos resumir los objetivos de la intervención de la siguiente forma:

1. Ayudar a Noelia y Manuel a constituirse como 
pareja y a adoptar el rol de padres.

2 . Contribuir a que establezcan una relación apropiada con las familias de origen.

3. Facilitar la relaciones de Noelia y Manuel con iguales.

4. Estimular el desarrollo personal y profesional de ambos y especialmente de Noelia.

1. Ayudarles a constituirse como pareja y adoptar el rol de padres. Quizás sea ésta la tarea más importante debido al salto de etapas que Noelia y Manuel han hecho y las dificultades de partida. Esto implica a su vez otras subtareas:

-En primer lugar, es necesario crear un límite claro como pareja lo que implica que ambos vivan juntos y con independencia de sus familias de origen.

-En segundo lugar, es importante negociar los acuerdos prácticos básicos que permita una vida en común.

-En tercer lugar, debemos mejorar la comunicación entre la pareja de modo que, por ejemplo permita negociar los desacuerdos sin sentirse deslegitimados ni culpables. Existe el peligro de que no se diga la verdad por miedo a herir, especialmente Manuel puede vivir esta situación ya que Noelia aparece como poco tolerante con las críticas que tiende a vivir (debido a su historia personal y a la dinámica de relación con su madre) como ataques personales.

-Finalmente, debemos definir los roles de ambos con respecto a la hija y conseguir un cuidado compartido de ésta. Como hemos dicho un peligro al que hacemos frente es que Noelia establezca una relación cerrada con su hija (se puede refugiar en ella, la única persona en la que puede confiar) desplazando a Manuel fuera. También es necesario que la hija no se convierta en un obstáculo a su intimidad como pareja. Esto no quiere decir que ahora no haya que respaldar la decisión de Noelia de lactar a la niña, ya que puede contribuir a que ésta sea capaz de hacer el vínculo afectivo madre hija que es tan necesario.

2. Contribuir a que establezcan una relación apropiada con las familias de origen. La necesaria separación de las familias de origen no debe hacerse de un modo traumático aunque es importante que tampoco se cree una dependencia excesiva. Hasta el momento esta oscilación entre dependencia y ruptura ha sido lo que ha caracterizado la relación de Noelia con la propia familia y la de Manuel. Evitar la ruptura con las familias de origen es un objetivo ya que siempre es positivo mantener lazos afectivos con ésta, para ellos y para la hija. Además la familias de ambos pueden ser una fuente de apoyo especialmente en situaciones críticas.

Indudablemente Noelia y Manuel van a necesi- tar apoyo de las familias de origen. La perspectiva en estos momentos es que sea la familia de Manuel la que esté mas preparada para hacerlo. Sin embargo, esto puede contribuir a mantener el actual desequilibrio en el balance de la relación en la pareja y que otra vez Noelia no sea capaz de aportar nada (solamente problemas). Por lo tanto, sería importante que Camino (madre de Noelia) pudiera cooperar. De hecho los pocos momentos de felicidad que muestra Camino se producen cuando se encarga de cuidar a su nieta. Hay que señalarle a Noelia que algo positivo ha tenido que recibir de Camino, cuando le ha capacitado para que ella sea capaz de formar una familia y de ser una madre competente. Pero al mismo tiempo hay que facilitar que Camino coopere sin resultar intrusiva con la nueva pareja y el hijo. Esto es particularmente difícil por la necesidad de ayuda para sí misma que tiene Camino, por su tendencia a mantener relaciones completamente absorbentes y a exigir fidelidades y dedicación absolutas. Podemos suponer que, de forma inconsciente, el proyecto de Camino pueda ser apartar a Manuel y cuidar con su hija a la nieta. Conseguir la necesaria flexibilidad por parte de Camino es uno de los retos más difíciles.

3. Facilitar que Noelia y Manuel tengan relaciones con iguales. Una de las razones por las que es necesario el apoyo de las familias de origen es que su contribución al cuidado de la hija permita que Noelia y Manuel no se queden encerrados en una relación sin vínculos sociales con jóvenes. La edad de ambos (Noelia 22 y Manuel 24) hace aconsejable que puedan mantener las diversiones propias de esa etapa vital. En caso contrario se puede crear frustraciones y resentimientos y provocarse tensiones en la pareja; sobre todo si uno de ellos mantiene una vida social propia (probablemente Manuel) mientras el otro, en este caso Noelia, se queda encerrada en casa.

4. Mantener el desarrollo personal y profesional de ambos y especialmente de Noelia. Noelia era una buena estudiante que terminó el BUP y el COU, pero su situación emocional en los últimos años le ha impedido continuar (estaba preparando la selectividad). Es necesario plantear el futuro laboral de Noelia después de los primeros meses de crianza. También queda pendiente valorar si Noelia necesita apoyo psicológico individual para reelaborar sus vivencias desde la muerte de su padre.

Todo este trabajo se debe realizar en entrevistas con la pareja, sin descartar sesiones individuales con Noelia no olvidándonos de que Camino, su madre, también necesita apoyo individual.

\section{EVOLUCIÓN}


Las primeras entrevistas con la pareja han permitido sosegar la situación y hacer que ambos se sitúen ante sus problemas de un modo más reposado. Se ha podido empezar a analizar algunos aspectos importantes, como es la decisión en torno a dónde vivir y las relaciones con las familias de origen. Ambos han decidido buscarse una casa para vivir independiente y no demasiado cerca de ninguna de las familias. La comunicación entre ambos miembros de la pareja es ahora mas fluida y Noelia se empieza a sentir más satisfecha consigo misma. Una vez llevado a cabo estos primeros pasos por medio de un contacto personal con el terapeuta de familia del centro de salud mental de referencia, se llevo a cabo la derivación de la pareja al nivel secundario. Esta medida fue debida, entre otras razones, a que el cambio de domicilio hacía difícil mantener la continuidad, a que el trabajo iba a ser largo y no exento de dificultades y finalmente a que el médico de familia quizás podía tener un papel protagonista en el apoyo a Camino (que es paciente suya desde hace tiempo).

\section{CONCLUSIONES}

Este caso es interesante para el médico de familia dado la presentación tan alarmante y la situación de partida inicialmente tan complicada de la pareja. Incluso en un primer momento al médico de familia le surgen dudas sobre la posibilidad de que Noelia sufra un trastorno psicopatológico grave de corte psicótico.

Sin embargo, las entrevistas con la pareja permiten contener la crisis y dirigir a la pareja, manteniendo un contacto con ellos, hacia el segundo nivel para un tratamiento más a largo plazo.

En la presentación de este caso también se ponen de relieve los peligros que representa la actual cultura psiquiátrica para el médico de familia. Un profesional puede poner algo tan importante a nivel psicológico como es la creación de un vínculo entre madre e hija (para lo cual la lactancia era importante) en un segundo plano frente a la posibilidad de prescribir un IRSS.

1. Vergeles Blanca J. Orientación Familiar en Atención Primaria de Salud. Pensar en términos de familia. Tri-

\section{CORRESPONDENCIA:}

José Ramón Loayssa Lara

Centro de Salud de Noain

Ctra. Vieja del Aeropuerto, s/n

31110 Noain

Navarra

\section{Bibliografía}

buna Docente en Medicina de Familia 2000; 1: 4: 438.

2. Gombrick-Graham L. A developmental model for Family Systems. Fam Process 1985; 24: 139-50.

3. Asen KE, Tomson P. Intervención familiar. Guía Práctica para los Profesionales de la Salud. Barcelona: Paidos, 1997.

4. Mc Goldrick M, Carter EA. The family life cycle. In: Walsh $F($ ed). Normal Family Processes. New York: Guilford Press, 1982.

5. Minuchin S. Family and family therapy. Cambridge: Harvard University Press, 1974.

6. Fernández Rojero C. Ciclo Vital y sistema familiar. Medifam 1991; 2: 94-8.

7. McDaniel S, Campbell TL, Seaburn DB. Orientación Familiar en Atención Primaria. Manual para médicos de atención primaria. Barcelona: Springer-Verlag lbérica, 1998; 119-38.

8. Jordan JR, Kraus DR, Ware ES. Observations on Loss and Family Development. Fam Proc 1993; 32: 425-40.

9. Recasens i Torras JM. La muerte en la Familia. Aten Primaria 1992; 10 : 562-5.

10. Jarabo Crespo Y. Familia, paciente terminal y duelo. Medifam 1994; 6: 301-4 .

11. Walsh F, Mcgoldrick M. La pérdida y el ciclo vital fami- liar. En: Falicov CJ. Transiciones de la familia. Continuidad y cambio en el ciclo de vida. Buenos Aires: Amorrortu Editores, 1991; 429-63.

12. Belart A, Ferrer M. El ciclo de la vida. Una visión sistémica de la familia. Bilbao: Desclée De Brouwer, 1999.

13. De los Rios A. El Nido sin usar. En: De la Revilla L. Manual de Atención Familiar. Granada: Fundación para el estudio de la Familia, 1999; 583-605.

14. Henderson J. The Many Roles of Fatherhood. Can Fam Physician 1992; 38: 629-37.

15. Roberts $\mathrm{L}$. The Family Life Cycle in Medical Practice. En: Crouch MA, Roberts L. The Family in Medical Practice. A Family Systems Primer. New York: Springer - Verlag, 1986; 74-96.

16. McDaniel S, Campbell TL, Seaburn DB. Orientación Familiar en Atención Primaria. Manual para médicos de atención primaria. Barcelona: Springer-Verlag lbérica, 1998; 296-309.

17. Bailon Muñoz E. La pareja que espera (Atención Familiar y Embarazo). En: De la Revilla L. Manual de Atención Familiar. Granada: Fundación para el estudio de la Familia, 1999, 607-32. 
